\title{
Delocalization induced by low-frequency driving in disordered tight-binding lattices
}

\author{
Dario F. Martinez and Rafael A. Molina \\ Max-Planck-Institut für Physik komplexer Systeme, Nöthnitzer Strasse 38, 01187 Dresden, Germany
}

(Received 11 January 2006; published 7 February 2006)

\begin{abstract}
We study the localization properties of disordered one-dimensional tight-binding lattices driven by ac fields. The localization length of the electrons increases when the frequency of the driving field is smaller than the bandwidth. We show that there is an optimal value of the amplitude of the driving field for which the localization length of the system is maximal. This maximum localization length increases with the inverse of the driving frequency.
\end{abstract}

DOI: 10.1103/PhysRevB.73.073104

PACS number(s): 72.15.Rn, 73.20.Fz, 73.21.Hb

Real materials always contain a certain degree of disorder since the atomic structure is never perfectly regular. In fact, many physical properties are either influenced or even mainly determined by this randomness. The understanding of the effects of disorder in the physical properties of a material is therefore of great practical importance and has played a central role in condensed matter physics in the last half century. ${ }^{1}$

One of the most simple disordered systems is the motion of a particle in a one-dimensional (1D) random potential. Realizations of this system can be studied experimentally, e.g., in semiconductor superlattices (SL) in the coherent regime. ${ }^{2}$ If the system is long enough its eigenstates are exponentially localized due to disorder. Electrons in these localized states are spatially confined and their only contribution to transport is through thermally activated hopping. The main quantity of interest in this case is the localization length $\lambda$ of the electron wave functions, which is controlled by the ratio between the bandwidth $\Delta$ and the strength of the disorder $W$. A disordered system of length $L>\lambda$ will behave as an insulator while a system with length $L<\lambda$ will behave as a conductor. ${ }^{1,3}$

The fascinating effects of radiation on the transport properties of SLs have been intensively studied both theoretically and experimentally in the last three decades. ${ }^{4}$ One of the most interesting effects, predicted by Dunlap and Kenkre, is dynamic localization (as defined in Ref. 5), also known as coherent destruction of tunneling, ${ }^{6}$ in which the electron wave function can be strongly localized in the presence of an ac electric field. This effect was shown to be associated with miniband collapse ${ }^{7}$ which occurs at the zeros of a Bessel function that depends on the field amplitude. At these points the width of the miniband goes to zero, the group velocity of a wave packet vanishes, and the electron becomes effectively localized. Experimentally, this effect was observed as a suppression of current at some amplitudes of the ac electric field. ${ }^{8}$

When a time-periodic driving is applied to a disorder tight-binding system, a new possibility emerges, i.e., controlling the localization of the system by changing the amplitude and/or frequency of the driving field. Holthaus et al. ${ }^{9}$ showed that for high frequencies, the Floquet states become localized (in contrast with dynamical localization) and their localization (participation ratio) depends on the ratio $\Delta_{e f f} / W$, where $\Delta_{e f f}=\Delta J_{0}(e V d / \hbar \omega)$. Here, $V$ and $\omega$ are the amplitude and frequency of the driving field and $d$ is the spatial period of the lattice.

In this paper we calculate the localization length of a driven disordered tight-binding lattice and we focus on the previously unexplored low frequency regime. For this purpose we use a Floquet-Green function formalism which makes use of matrix continued fractions. ${ }^{10}$ This formalism allows us to generalize the definition of $\lambda$ for time-periodic systems and to calculate its value. We found that there are two distinct and clearcut regimes in this system: the highfrequency regime for $\hbar \omega>(\Delta+W) / 2$ and the low-frequency regime for $\hbar \omega<(\Delta+W) / 2$. In the first one, we found that $\lambda$ is a function of $\Delta_{e f f} / W$, in perfect agreement with previous works. ${ }^{9}$ In this regime, the driving field always contributes to localize even more the wave function (as compared to the nondriven case). In contrast, in the low-frequency regime, we find that the driving can significantly delocalize the electrons. In addition to this new result, we provide an intuitive explanation of why this should be so: For low frequency, each additional Floquet channel created by the driving provides the electron with new paths which will differ in their degree of localization, with some having smaller and others having greater localization length as compared to the nondriven case. Since $\lambda$ is determined only by the path with the greatest localization length, after the ensemble average has been performed, additional propagation channels should always contribute to increase $\lambda$. The same situation does not occur in the high-frequency case because the additional paths introduced by the absorbtion or emission of one or more photons always have localization lengths smaller than in the nondriven case.

We will study the single-band Anderson Hamiltonian with diagonal disorder ${ }^{11}$ plus a time-periodic potential,

$$
\begin{aligned}
H= & -\frac{\Delta}{4} \sum_{j}(|j+1\rangle\langle j|+| j\rangle\langle j+1|) \\
& +\sum_{j} \epsilon_{j}|j\rangle\left\langle j\left|+2 V \cos \omega t \sum_{j}\right| j\right\rangle j\langle j| .
\end{aligned}
$$

The on-site energies $\epsilon_{j}$ are distributed uniformly from $-W / 2$ to $W / 2$. The driving potential is due to an ac electric field of amplitude $2 V$. (The factor of 2 is for convenience.) In a SL this term can represent $\mathrm{THz}$ radiation linearly polarized in the growth direction of the lattice. The quantity $\Delta$ is equal to 
the bandwidth of the nondriven system without disorder. We will use units in which $\hbar=1$ and we set $\Delta=4$.

For a system that obeys discrete time-translational symmetry with period $T$, there exists a complete set of solutions to the Schrödinger equation of the form $\left|\Psi^{\alpha, p}(t)\right\rangle$ $=\exp \left(-i e_{\alpha, p} t / \hbar\right)\left|\phi^{\alpha, p}(t)\right\rangle$, where $\left|\phi^{\alpha, p}(t)\right\rangle=\left|\phi^{\alpha, p}(t+T)\right\rangle$. These are called the Floquet states of the system and the periodic functions $\left|\phi^{\alpha, p}(t)\right\rangle$ obey an eigenvalue equation similar to the static Schrödinger equation,

$$
\left[H(t)-i \hbar \frac{d}{d t}\right]\left|\phi^{\alpha, p}(t)\right\rangle=e_{\alpha, p}\left|\phi^{\alpha, p}(t)\right\rangle,
$$

with $H(t)$ the time-periodic Hamiltonian of the system. The eigenvalues of this equation can be written as $e_{\alpha, p}=\epsilon_{\alpha}$ $+p \hbar \omega$, for $0 \leqslant \epsilon_{\alpha} \leqslant \hbar \omega$ and $p$ an integer. A Floquet-Green operator corresponding to Eq. (2) can be defined and its Fourier components satisfy

$$
G^{(k)}(E)=\sum_{\alpha, p} \frac{\left|\phi_{k+p}^{\alpha, 0}\right\rangle\left\langle\phi_{p}^{\alpha, 0}\right|}{E-\epsilon_{\alpha}-p \hbar \omega}
$$

where $\left|\phi_{p}^{\alpha, 0}\right\rangle$ are the Fourier components of the Floquet eigenfunctions $\left|\phi^{\alpha, 0}\right\rangle=\Sigma_{p} e^{-i p \omega t}\left|\phi_{p}^{\alpha, 0}\right\rangle$. The transport properties of driven systems have been formulated in terms of this Floquet-Green operator which plays a similar role to the Green operator in the Landauer formalism for conduction. ${ }^{12}$ In the limit $V \rightarrow 0$, one can show that $G^{(0)}(E)$ becomes the usual Green function of a time-independent system and that $G^{(k \neq 0)}(E)=0$. The well-known definition of localization length, in terms of Green functions for nondriven systems, ${ }^{3}$ can be generalized according to

$$
\frac{1}{\lambda^{(k)}(E)}=-\lim _{L \rightarrow \infty} \frac{1}{L}\left\langle\ln \left|G_{1 L}^{(k)}(E)\right|\right\rangle .
$$

The different quantities $G_{1 L}^{(k)}(E)$ are associated with the probability of a process where an electron starts with an energy $E$ at site 1 and ends at site $L$ with energy $E+k \hbar \omega$. Each one of these processes in principle can have a different localization length associated to it. However, assuming that the asymptotic behavior of the Floquet eigenstates is exponentially decreasing, one can see that in the limit $L \rightarrow \infty$, the dominant term in the sum over $p$ in Eq. (3) will always be the same ( $p$ can be positive or negative), independently of the value of $k$. This implies that all the quantities $G_{1 L}^{(k)}(E)$ decay at the same rate with $L$ (even though their values can be very different, depending on $V$ and $\omega$ ). Consequently, the quantities $\lambda^{(k)}(E)$ are all identical. From now on we will only compute $\lambda(E) \equiv \lambda^{(0)}(E)$.

For the calculation of $G^{(0)}(E)$ we use a method developed by one of the authors (see Ref. 10 for details). We want to calculate the Floquet-Green operator for a periodic Hamiltonian of the form $H(t)=H_{0}+2 \cos (\omega t) \hat{V}$, where $H_{0}$ and $\hat{V}$ are any time-independent operators in the Hilbert space of the system. The Floquet components of the Green operator for this Hamiltonian satisfy,



FIG. 1. (Color online) $\left\langle\ln G_{1 L}(0)\right\rangle$ as a function of the length of the system $L$ for different values of disorder $W$ and frequency $\omega$. High-frequency results, $\omega=20$, show regular oscillations on top of the exponential decay, according to the equation $G_{1 L}(0)$ $=A J_{0}(2 V L / \hbar \omega) \exp ^{-L / \lambda}$. Low frequency curves do not show these oscillations. The value of $\lambda$ is the negative inverse slope of the linear fits. The ensemble average was made with 1000 different realizations of disorder.

$$
\left(E+k \hbar \omega-H_{0}\right) G^{(k)}-\hat{V}\left(G^{(k+1)}+G^{(k-1)}\right)=\delta_{k, 0} .
$$

These equations can be solved using matrix continued fractions. For the case $k=0$, one gets

$$
G^{(0)}(E)=\left[E-H_{0}-\hat{V}_{e f f}(E)\right]^{-1},
$$

where

$$
\hat{V}_{e f f}=\hat{V}_{e f f}^{+}(E)+\hat{V}_{e f f}(E)
$$

with

$$
\hat{V}_{e f f}^{ \pm}(E)=\hat{V} \frac{1}{E \pm 1 \hbar \omega-H_{0}-\hat{V} \frac{1}{E \pm 2 \hbar \omega-H_{0}-\hat{V} \frac{1}{\vdots} \hat{V}} \hat{V}} \hat{V} .
$$

The convergence of Eq. (8) is system specific. For our Hamiltonian, Eq. (1), the number of bands necessary to ensure convergence increases linearly with $V L / \omega$. The numerical performance of our method is determined by the speed in the calculation of an $L \times L$-matrix inverse for each Floquet sideband. For our disordered system we know that the localization length in the middle of the band $(E=0)$ for $V=0$ behaves as $\lambda_{0} \approx 105[\Delta /(4 W)]^{2} .{ }^{13}$ In this case it is also known that $\lambda$ as a function of the energy $E$ follows a parabolic law with a maximum at $E=0$. For $|E|>(\Delta+W) / 2$ the localization length decreases rapidly with $|E|$. In this work, we will always take $E=0$.

In Fig. 1 we show some examples of the results obtained for the ensemble average of $\ln G_{1 L}(0)$ as a function of the length of the system $L$. (We have dropped the superscript in 


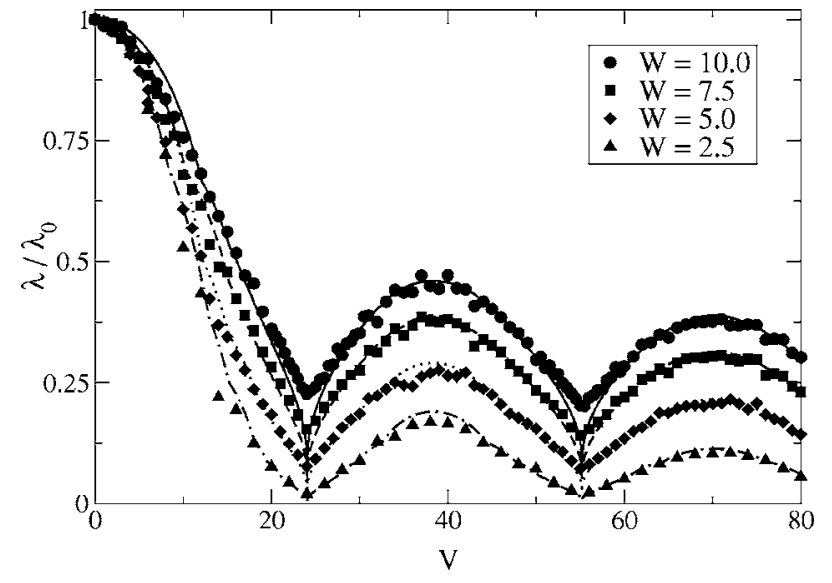

FIG. 2. High-frequency results. Results for different values of disorder $W$ with $\omega=20.0$. The corresponding results for a nondriven system with renormalized bandwidth are shown with lines (obtained from numerical data).

the Floquet-Green operator.) As expected, a straight line fits the data very well. The wave functions decay exponentially with the distance. The negative slope of these curves corresponds to the inverse of the localization length when $L \gg \lambda$. It is important to point out that the deviations from this exponential behavior are not due to a deficient ensemble average. For high frequencies they show a regular pattern, a manifestation of dynamic localization which shuts off the tunneling between the sites $L-1$ and $L$ at the zeros of $J_{0}(2 V L / \omega)$. In fact, for high frequency and $L \gg \lambda$ one can show that the Floquet-Green function can be expressed analytically as $G_{1 L}(0)=A J_{0}(2 V L / \omega) \exp (-L / \lambda)$, where $A$ is a constant independent of $L$. As shown in Fig. 1 for the second and last sets of data (from top to bottom), the thin (red online) continuous line representing this analytical result fits the numerical data very well. For low frequencies, the Green function also decays exponentially. However, the deviations from this behavior cannot be expressed analytically and seem to occur equally on both sides of the straight-line fit.

We now show results for the high-frequency regime, which has been discussed in the literature, ${ }^{9}$ although to the best of our knowledge, no calculation of $\lambda$ has been reported. The high-frequency limit can be characterized as the regime in which the absorption or emission of any number of photons would leave the particle with an energy outside of the region where the eigenenergies of the nondriven system concentrate. This region is well known to have a width $\Delta+W$, and therefore, at the center of the band this condition is satisfied when $\omega>(\Delta+W) / 2$. In the high-frequency regime, the results for $\lambda$ are obtainable from the function $\lambda_{0}(\Delta / W)$ but with a renormalized hopping term $\Delta \rightarrow \Delta J_{0}(2 V / \omega)$. Figure 2 shows, for $\omega=20.0$ and for several different values of disorder, that the numerical data is in excellent agreement with Holthaus' result. The minima of $\lambda$ correspond to the zeros of the Bessel function $J_{0}(2 V / \omega)$.

In Fig. 3 we show the localization length $\lambda$ as a function of $V$ for low and high frequencies and for two values of disorder, $W=10.0$ (top) and $W=5.0$ (bottom). Here, one can see that there is a fundamental difference between those two
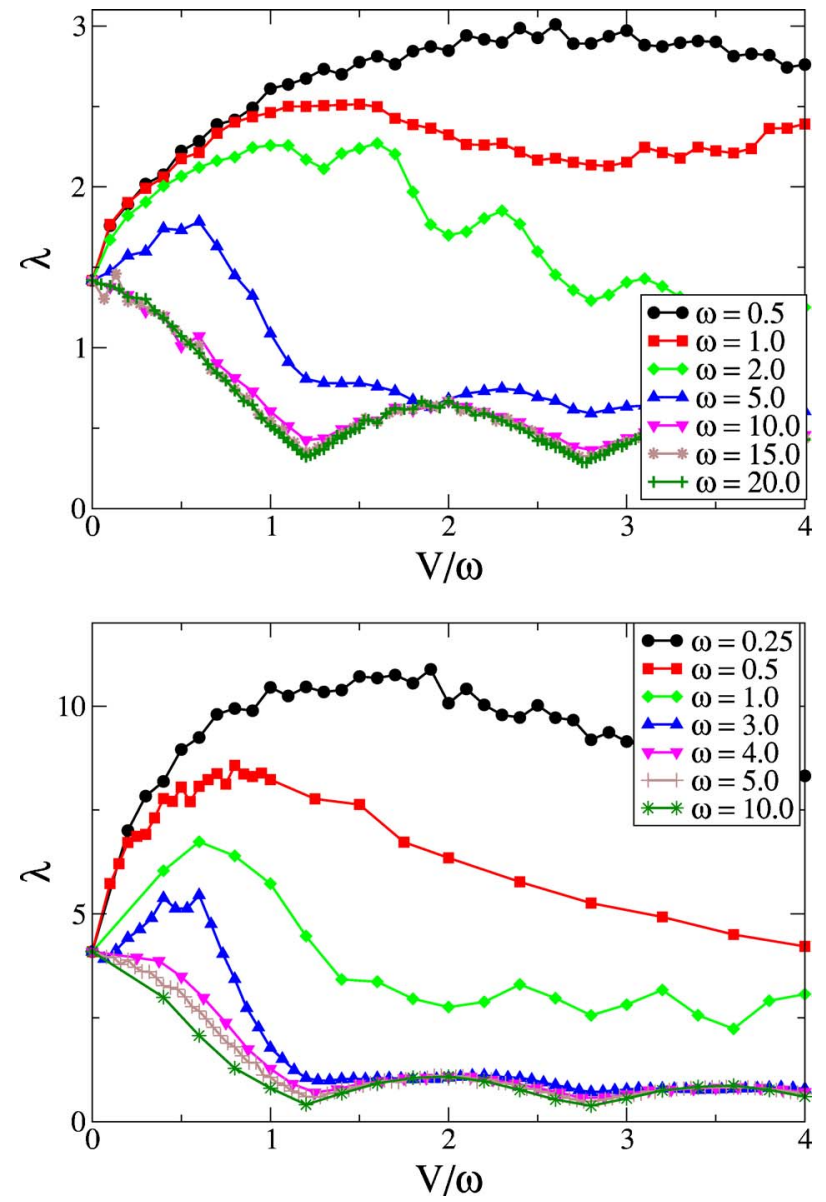

FIG. 3. (Color online) $\lambda$ as a function of $V / \omega$ for $W=10$ (top panel) and $W=5$ (bottom panel) and different values of $\omega$.

regimes: For $\omega<(\Delta+W) / 2$ and $V$ small, $\lambda$ increases with $V$, whereas for $\omega>(\Delta+W) / 2$, it decreases. This delocalization of the wave function due to low-frequency driving is the main result of this work. For all the frequencies that we explored in the low-frequency regime, as $V$ was increased $\lambda$ initially increased, then reached a maximum, and finally decreased (with some oscillations). This behavior can intuitively be understood if one assumes that the driving provides the electron with new propagating channels $n$, each one with a different localization length $\lambda_{i}$. According to this, one expects $\lambda=\max \left\{\lambda_{1}, \lambda_{2}, \ldots, \lambda_{n}\right\}$. Given the linear dependence of the total number of Floquet sidebands with $V$, it is expected that $n \propto V$, and therefore $\lambda$ should initially increase with $V$. This is valid until we reach the maximum number of effective channels that can be supported in the region of the spectrum where the localization lengths are comparable (size $\approx \Delta+W)$. Here, $\lambda$ reaches a maximum value $\lambda_{\max }$. Beyond this point, $\lambda$ decreases with $V$ (with oscillations), as the weight of the Floquet eigenstates oscillates and shifts to energies outside the band.

In Fig. 4 we plot the data for $\left(\lambda_{\max }-\lambda_{0}\right) / \lambda_{0}$ vs $1 / \omega$. This figure suggests that low-frequency driving is more effective in delocalizing a weakly disordered system than a strongly disordered one. The continuous curves correspond to the fit of a very simple mathematical model where the maximum number of propagating channels is inversely proportional to 




FIG. 4. (Color online) Behavior of $\lambda_{\max }$ as a function of $1 / \omega$ for different values of disorder $W$. The numerical data have been fitted using a function $\lambda\left(n_{\max }, \sigma\right)$ derived from a simple statistical model where the maximum number of effective channels is inversely proportional to the frequency, $n_{\max }=c / \omega$ and $\sigma$ is the standard deviation of each channel (with exponential probability function).

the frequency $n_{\max }=c / \omega$. We also assume that each one these channels has an exponential distribution function with standard deviation $\sigma$ (a Gaussian distribution gives similar results). We can see that this simple model fits the data very well. Despite the fact that some of the assumptions in this simple model are not formally justified, we believe that its agreement with many of the features in the numerical data support our intuitive explanation for the delocalization of the wave function at low frequencies. In our system, $\lambda_{\max }$ seems to increase monotonically with the inverse of $\omega$. However, in a real system a limiting time scale might appear, i.e., the energy-relaxation time $\tau$. (In real systems, this $\tau$ can be controlled reducing the temperature.) When $\omega<1 / \tau$ the delocalization induced by the driving field is limited and the curves shown on Fig. 4 will bend downwards. In the adiabatic limit $\omega \rightarrow 0, \lambda$ can be calculated by the time average over one cycle of the Green function of the stationary system. ${ }^{14}$ We have checked that our numerical results coincide with this time average when we use $\tau \neq 0$ and $\omega<1 / \tau .{ }^{15}$ For a disordered SL of length $L \approx \lambda_{0}$ a change of several orders of magnitude in the dynamic conductivity should be observed when going from frequencies higher than the bandwidth to smaller frequencies. Also, the behavior of the transport properties with temperature should be very different, from a hopping regime with some activation energy which is favored by higher temperatures to a normal regime where transport processes should be hindered by phonons and other temperature related effects. Other systems can also be used to observe these delocalizing effects, e.g., atom optical traps, which have been proposed as a testing ground of Anderson localization. For this system, the lattice can be implemented by far detuned counterpropagating laser beams, the ac driving can be obtained using a periodic phase shift between the beams, ${ }^{16}$ and the random potential can be obtained with a superimposed random speckle pattern. ${ }^{17}$

In this work we have calculated the localization length for a one-dimensional Anderson model in a homogeneous ac electric field. We have found two very different regimes according to whether the frequency of the driving is smaller or bigger than the bandwidth. For low-frequency driving, the localization length increases for moderate values of the driving amplitude, while for high frequency the localization length decreases due to the Bessel function renormalization of the miniband width. We have shown that low-frequency driving can increase the localization length by providing new propagation channels. In this sense, the effect of such a field can be compared to an increase in the dimensionality of the system, which suggests that, in disordered systems where a metal-insulator transition is expected when the dimension of the system increases, low-frequency driving could have very dramatic delocalizing effects.

The authors would like to thank H. Schomerus, G. Platero, R. A. Jalabert, and D. Weinmann for their suggestions regarding the presentation of this manuscript.
${ }^{1}$ I. M. Lifshits, S. A. Gredeskul, and L. A. Pastur, Introduction to the Theory of Disordered Systems (John Wiley and Sons, New York, 1988).

${ }^{2}$ L. Esaki and R. Tsu, IBM J. Res. Dev. 14, 61 (1970).

${ }^{3}$ B. Kramer and A. MacKinnon, Rep. Prog. Phys. 56, 1469 (1993).

${ }^{4}$ G. Platero and R. Aguado, Phys. Rep. 395, 1 (2004).

${ }^{5}$ D. H. Dunlap and V. M. Kenkre, Phys. Rev. B 34, 3625 (1986).

${ }^{6}$ F. Grossmann, T. Dittrich, P. Jung, and P. Hänggi, Phys. Rev. Lett. 67, 516 (1991).

${ }^{7}$ M. Holthaus, Phys. Rev. Lett. 69, 351 (1992).

${ }^{8}$ B. J. Keay, S. Zeuner, S. J. Allen, Jr., K. D. Maranowski, A. C. Gossard, U. Bhattacharya, and M. J. W. Rodwell, Phys. Rev. Lett. 75, 4102 (1995).

${ }^{9}$ M. Holthaus, G. H. Ristow, and D. W. Hone, Phys. Rev. Lett. 75, 3914 (1995); M. Holthaus and D. W. Hone, Philos. Mag. B 74,
105 (1996)

${ }^{10}$ D. F. Martinez, J. Phys. A 36, 9827 (2003); 38, 1 (2005).

${ }^{11}$ P. W. Anderson, Phys. Rev. 109, 1492 (1958).

${ }^{12}$ S. Kohler, J. Lehmann, and P. Hänggi, Phys. Rep. 406, 379 (2005).

${ }^{13}$ G. Czycholl, B. Kramer, and A. MacKinnon, Z. Phys. B: Condens. Matter 43, 5 (1981); M. Kappus and F. Wegner, ibid. 45, 15 (1981).

${ }^{14}$ M. Moskalets and M. Büttiker, Phys. Rev. B 66, 205320 (2002).

${ }^{15}$ D. F. Martinez and R. A. Molina (unpublished).

${ }^{16}$ K. W. Madison, M. C. Fischer, R. B. Diener, Qian Niu, and M. G. Raizen, Phys. Rev. Lett. 81, 5093 (1998).

${ }^{17}$ P. Horak, J.-Y. Courtois, and G. Grynberg, Phys. Rev. A 58, 3953 (2000). 